\title{
E-region echo characteristics governed by auroral arc electrodynamics
}

\author{
S. E. Milan ${ }^{1}$, N. Sato ${ }^{2}$, M. Lester ${ }^{1}$, Y. Murata ${ }^{3}$, Y. Shinkai ${ }^{3}$, H. Doi ${ }^{4}$, H. U. Frey ${ }^{5}$, and T. Saemundsson ${ }^{6}$ \\ ${ }^{1}$ Department of Physics and Astronomy, University of Leicester, Leicester LE1 7RH, UK \\ ${ }^{2}$ National Institute of Polar Research, Tokyo 173-8515, Japan \\ ${ }^{3}$ Graduate University for Advanced Studies, Tokyo 173-8515, Japan \\ ${ }^{4}$ Tokai University, Kanagawa 259-1292, Japan \\ ${ }^{5}$ Space Sciences Laboratory, University of California at Berkeley, California, USA \\ ${ }^{6}$ Science Institute, University of Iceland, Reykjavik IS-107, Iceland
}

Received: 23 October 2002 - Accepted: 13 February 2003

\begin{abstract}
Observations of a pair of auroral arc features by two imagers, one ground- and one space-based, allows the associated field-aligned current (FAC) and electric field structure to be inferred. Simultaneous observations of HF radar echoes provide an insight into the irregularitygenerating mechanisms. This is especially interesting for the E-region echoes observed, which form the focus of our analysis, and from which several conclusions can be drawn, summarized as follows. Latitudinal variations in echo characteristics are governed by the FAC and electric field background. Particularly sharp boundaries are found at the edges of auroral arcs. Within regions of auroral luminosity, echoes have Doppler shifts below the ion-acoustic speed and are proportional to the electric field, suggesting scatter from gradient drift waves. Regions of downward FAC are associated with mixed high and low Doppler shift echoes. The high Doppler shift component is greatly in excess of the ionacoustic speed, but seems to be commensurate with the driving electric field. The low Doppler shift component appears to be much depressed below expectations.
\end{abstract}

Key words. Ionosphere (ionospheric irregularities; electric fields and currents)

\section{Introduction}

Previous studies have demonstrated the existence of a number of echo types in HF radar backscatter from the E-region (Villain et al., 1987, 1990; Hanuise et al., 1991; Milan and Lester, 2001; Milan et al., 2003) and have also investigated the relationship between E-region backscatter and visual auroral forms (Milan et al., 2000, 2001, 2002). In this study we present observations of an auroral feature made from above and below, that is by a ground- and a space-based imager simultaneously, and show the remarkable association with HF radar E-region echoes. Not only does this allow the associ-

Correspondence to: S. E. Milan

(Steve.Milan@ion.le.ac.uk) ation between echo types and auroral forms (precipitation) to be elucidated, but it also reveals the relationship between electric field and echo characteristics, as we are able to approximate the electrodynamics of the arc system under investigation.

\section{Experimental arrangement}

The CUTLASS Iceland HF radar, which forms part of the international SuperDARN radar network (Greenwald et al., 1995), has been used extensively to study the characteristics of E-region backscatter, due to its favourable viewing geometry. In such studies, the observing mode is optimized for E-region investigation by reducing the gate length to a minimum and concentrating soundings to ranges close to the radar. This "myopic" configuration has been described in detail previously (e.g. Milan and Lester, 2001), so here we just summarize the key points. The radar sounds 16 beams in sequence, taking approximately $1 \mathrm{~min}$ to complete a scan. Each beam is gated into 75 cells of $15 \mathrm{~km}$ in length, with a range to the first gate of $180 \mathrm{~km}$; hence, the maximum sounded range is $1305 \mathrm{~km}$. The radar location and field-of-view are shown in Fig. 1. Routine analysis of the obtained auto-correlation functions provides backscatter power, spectral width, and Doppler shift of the echo spectra, and a measure of the elevation angle of the incoming signals, from which the altitude of the scattering volume can be estimated. In addition, the "stereo" upgrade of the radar undertaken in late 2001 allows the radar to sound at two different frequencies simultaneously. The "stereo myopic" mode, which was operating during the period of study, transmits continuously at $10 \mathrm{MHz}$, while also cycling through $8,12,14,16$ and $18 \mathrm{MHz}$ in consecutive scans, as described by Milan et al. (2003). In this paper, we concentrate mainly on the $10-\mathrm{MHz}$ observations.

The myopic operation of the radar was run in conjunction with a white-light all-sky camera (ASC) campaign conducted at three observing sites in Iceland, Raufarhofn, Tjörnes, and Grimsstadir, all shown in Fig. 1. The ASC at Raufarhofn, 


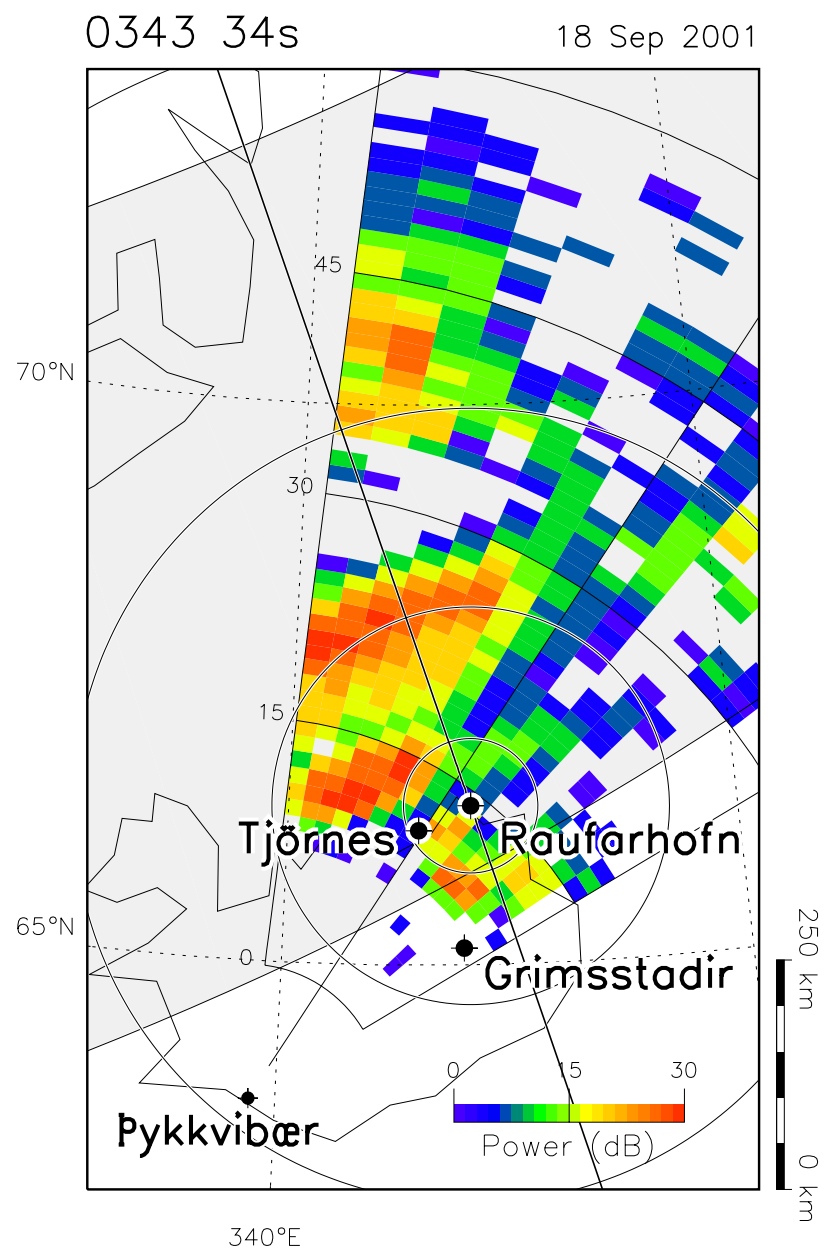

Fig. 1. A map showing the vicinity of Iceland and southeastern Greenland, indicating the location of the Pykkvibær radar and the three all-sky camera sites. The radar field-of-view is mapped assuming a backscatter altitude of $110 \mathrm{~km}$. Colour-coded is the backscatter power from the radar scan starting 03:43:34 UT on 18 September 2001. The projection of the Raufarhofn ASC field-ofview is shown, the four concentric circles marking the locii of zenith angles of $30^{\circ}, 60^{\circ}, 75^{\circ}$ and $85^{\circ}$ mapped to an altitude of $110 \mathrm{~km}$. The geomagnetic meridian employed to produce keograms is also shown. Superimposed is the statistical auroral oval for a $K_{p}$ level of 3 .

as the most northerly of the trio, was best placed for the auroral conditions experienced during the interval of interest, and it is this imager on which we focus our attention. Comparison of the radar and auroral observations was achieved using the method described by Milan et al. (2002). Thus, we produced a keogram of the ASC measurements along the geomagnetic meridian passing through the zenith of the ASC field-of-view. This meridian is indicated in Fig. 1, along with the locii of zenith angles $30^{\circ}, 60^{\circ}, 75^{\circ}$ and $85^{\circ}$. By mapping the ASC meridian into the radar field-of-view to an altitude of $110 \mathrm{~km}$ we are also able to produce "radar keograms" to allow for a direct comparison between the auroral and echo characteristics.

Finally, to allow for a more global overview of the auro- ral configuration present during the interval of interest we employed images from the Far Ultraviolet Wideband Imaging Camera (FUV/WIC) on board the IMAGE spacecraft (Mende et al., 2000a, b). These images were obtained every $2 \mathrm{~min}$, with an exposure time of $10 \mathrm{~s}$. The imager is sensitive to emissions in the FUV wavelength band 140-190 nm, which comprises Lyman-Birge-Hopfield (LBH) lines produced by electron impact excitation of $\mathrm{N}_{2}$.

\section{Observations}

On the night of 18 September 2001, the clouds parted for a brief interval between 02:00 and 04:00 UT. It is the auroral and radar observations from this period that we investigate in this study. Figure 1 shows the backscatter power recorded by the radar during the scan starting at 03:43:34 UT. In the near-range portion of the field-of-view (closer than range gate 30), several high power features are seen to lie along the L-shell direction, that is parallel to the edges of the statistical oval, shown for a $K_{p}$ of 3. Such L-shell-aligned features are characteristic of E-region backscatter (e.g. Milan and Lester, 2001), and indeed, measurements of the elevation angles of the echoes indicate that they originate from E-region altitudes. At further ranges, echoes are received from the F-region, and here, L-shell-aligned features are not so obvious.

Figure 2 shows three radar keograms (panels (a-c)) from the ASC meridian shown in Fig. 1, indicating backscatter power, Doppler shift, and spectral width, respectively, and the ASC keogram (panel (d)), from the period 02:45 to 04:00 UT. By their very nature, keograms are plotted as a function of zenith angle along the ASC meridian. This is ideal for comparison of the radar and auroral observations, but does tend to squash features at high zenith angles. To allow for a less spatially-distorted view of the radar observations, panel (e) shows the Doppler shift measurements as a function of range along beam 3 of the radar; the range here is the latitudinal distance from the radar.

First, we describe the auroral observations (Fig. 2d). Very little luminosity is observed along the meridian prior to 03:00 UT. After this time an auroral feature appears near a zenith angle of $45^{\circ}$, and subsequently progresses equatorwards over the zenith of the camera and eventually broadens to cover the zenith angle range $-50^{\circ}$ to $30^{\circ}$. Simultaneously, a second auroral feature appears polewards of the first near 03:05 UT, at a zenith angle of $65^{\circ}$, and then moves further polewards to a zenith angle of $80^{\circ}$. In other words, around 03:00 UT the auroral oval (which we will demonstrate corresponds to the first luminosity feature) brightens and expands equatorwards as a second arc forms polewards of it. These two features are roughly parallel and L-shell aligned, as demonstrated by the ASC snapshot image from 03:44 UT shown in Fig. 3a. Superimposed is the projection of the radar field-of-view; a vertical cut through the zenith of the image represents the magnetic meridian from which the keograms are formed. For the purposes of the present study we can con- 


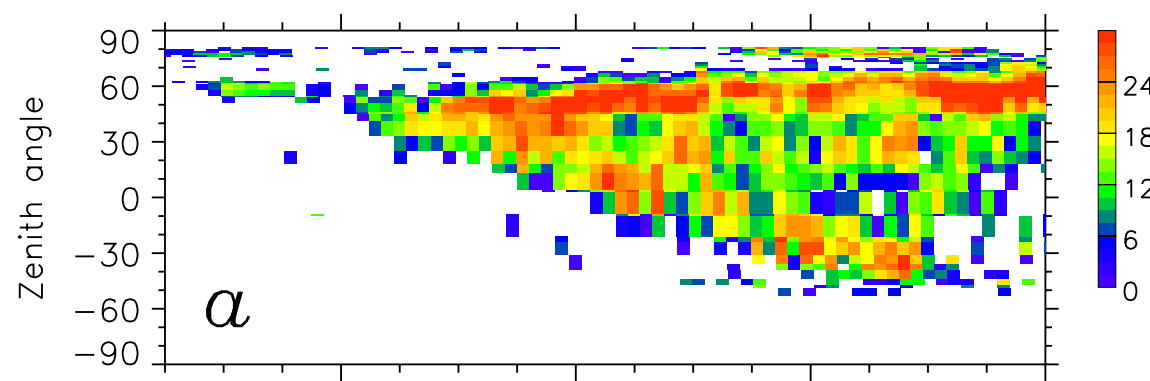

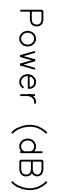
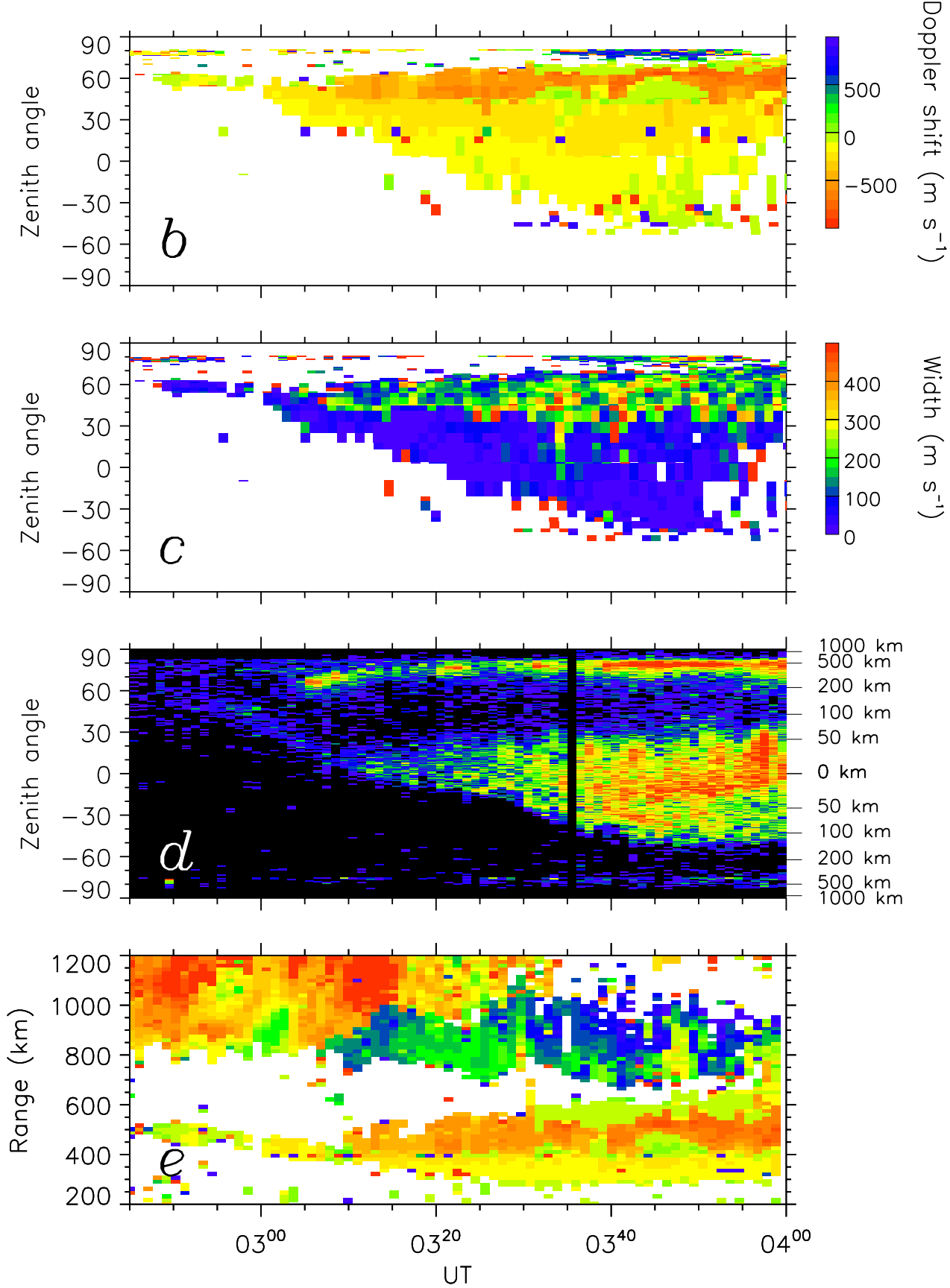

Fig. 2. (a - c) Radar keograms of backscatter power, Doppler shift (negative away from the radar), and spectral width, for the interval 02:45 to 04:00 UT. (d) The corresponding Raufarhofn ASC keogram. (e) A range-time-Doppler shift plot from beam 3 of the radar. Here, range corresponds to the latitudinal distance from the radar. 

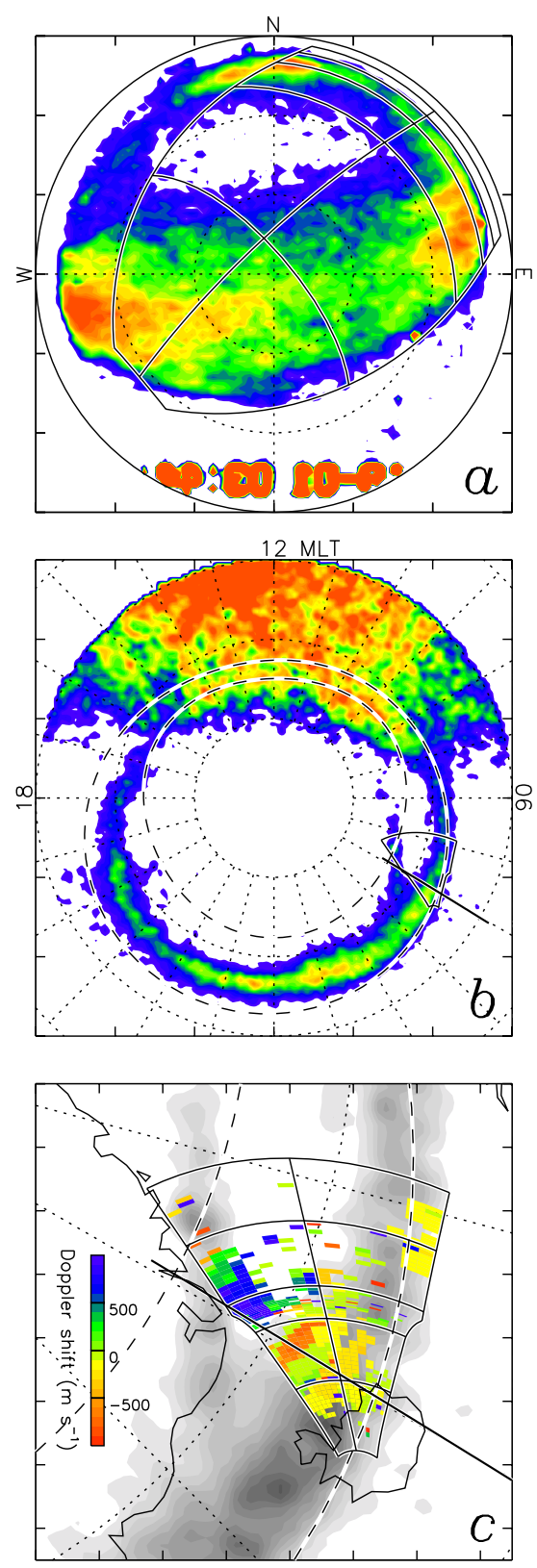

Fig. 3. Near simultaneous observations of the aurora from the ground and space, together with concurrent radar measurements, all from near 03:44 UT. (a) ASC image from 03:44:00 UT, aligned with the magnetic cardinal points. The north-south line is the magnetic meridian from which keograms are formed. Also shown is the projection of the radar field-of-view. Red features at the bottom of the image are timing information. (b) An auroral image from the IMAGE FUV/WIC instrument, taken at 03:44:34 UT. Superimposed is the $K_{p}=3$ statistical oval (dashed lines), the ASC meridian, and the radar field-of-view. (c) The relationship between Doppler shift, observed by the radar during the scan starting 03:43:34 UT, and auroral luminosity as measured by IMAGE. In the radar field-of-view every 15 th range gate is marked. The kink in the field-of-view between range gates 30 and 45 corresponds to a change in projection altitude from the E-region (nearer ranges) to the F-region. sider the luminosity features to be an arc-pair, though using the term "arc" in a fairly loose way. Auroral arcs, produced by precipitating electrons, are often associated with sheets of upward field-aligned current (FAC), and we will show below that the radar flow observations are consistent with this, especially if the gap between the arc pair is considered to be a sheet of downward return current. We will refer to three regions, from south to north, as arc 1, gap, and arc 2 .

Turning to the radar observations in panels (a-c) of Fig. 2, we immediately note the remarkable correspondence between the location of backscatter echoes and the visual aurora shown in panel (d). Specifically, significant radar backscatter only appears after 03:00 UT, starting near a zenith angle of $50^{\circ}$, though subsequently expanding polewards and equatorwards to cover a similar range of zenith angles as the visual arcs. (We note that no backscatter can be observed above a zenith angle of $83^{\circ}$, as here the ASC meridian falls outside the radar field-of-view.) There are discrepancies, however. For instance, backscatter is observed where the gap between the arc-pair lies, though the spectral characteristics of these echoes obviously differ considerably from the echoes associated with either arc. We describe these characteristics now in latitudinal order.

Associated with arc 1, echoes have Doppler shifts which tend to be low and negative (away from the radar), between 0 and $-300 \mathrm{~m} \mathrm{~s}^{-1}$, though increasing slightly in magnitude with latitude. These echoes have variable backscatter powers, though mainly below $30 \mathrm{~dB}$, and low spectral widths, less than $100 \mathrm{~m} \mathrm{~s}^{-1}$. Associated with the gap, backscatter power is enhanced to around $30 \mathrm{~dB}$, and the spectral widths tend to be higher, though variable, above $100 \mathrm{~m} \mathrm{~s}^{-1}$. These echoes display a mixture of high $\left(-500 \mathrm{~m} \mathrm{~s}^{-1}\right)$ and low (near $0 \mathrm{~m}$ $\mathrm{s}^{-1}$ ) Doppler shifts, with few intermediate values. Close examination of the echoes in this region shows that these mixed echoes are the "two-peak E-region echoes" or TWOPEE backscatter population identified by Milan et al. (2003). Such backscatter is thought to result from a pair of scattering layers within the E-region (high and low Doppler shifts from higher and lower in altitude, respectively) in which different irregularity types exist. The high, variable spectral widths occur as a consequence of two components, with high and low Doppler shift, co-existing in the echo spectra. The high Doppler shift component is found close to the line-of-sight component of the electron drift, whereas the low Doppler shift component is depressed significantly below this, approximately $10 \%$ of the line-of-sight component of the electron drift. The present observations suggest that such echoes are found not co-located with auroral forms, but adjacent to them, indeed potentially in regions of downward FAC.

The backscatter associated with both regions described above, arc 1 and the gap, is shown by elevation angle measurements to originate in the E-region ionosphere. The backscatter associated with arc 2, however, is of F-region origin, being further from the radar. As a consequence, this backscatter cannot tell us anything about E-region irregularity characteristics, but is still of interest as it reveals information regarding the electrodynamics of the arc-pair. These 
echoes only appear in the radar keograms between 03:30 and 03:55 UT (zenith angles greater than $75^{\circ}$ ), but are actually present throughout the interval of study (Fig. 2e). These echoes have positive Doppler shifts (towards the radar) and hence, correspond to plasma drift in the opposite direction to that in the lower latitude backscatter regions. Since this scatter lies at very high zenith angles, where features are significantly distorted in the keograms, it is difficult to determine the exact relationship with arc 2 . Global imagery shown below demonstrates that these echoes appear just equatorward of arc 2, not co-located with it. In other words, these echoes are also associated with the arc-pair gap. In the distorting keograms the latitudinal extent of this region of backscatter appears limited, but Fig. 2e reveals that it is just as extensive, if not more so, than the lower latitude gap backscatter.

Figure $3 b$ puts these observations into a more global context, being an IMAGE FUV/WIC snapshot from 03:44:34 UT, presented in a magnetic latitude and local time coordinate system. Overlaid is a statistical auroral oval, the location of the radar field-of-view, and the ASC keogram meridian. Iceland is located near 04 MLT at this time. In the image the auroral oval is clearly evident, though somewhat obscured by dayglow near noon. In addition, in the morning sector, an arc poleward of the main oval is seen, connected to the oval between 03 and 04 MLT, and extending around at least as far as 06 MLT and possibly to 07 MLT. In this image we can draw a clear association between arc 1 in the ASC observation and the main oval, and between arc 2 and the poleward auroral feature, as described previously. Figure $3 \mathrm{c}$ zooms in to the vicinity of the radar field-of-view, and overlays the concurrent radar measurements of Doppler shift (scan starting 03:43:34 UT). This places the radar observations somewhat to the east of the fork in the auroral structure, with the high positive Doppler shifts equatorward of arc 2 . In addition, there is a limited region of smaller positive Doppler shifts co-located with this arc. In the vicinity of the main oval, high negative Doppler shifts can be seen at the poleward edge of the luminosity, and co-located with it smaller negative velocity echoes.

\section{Discussion}

The CUTLASS Iceland radar has proved important for the study of backscatter echoes from decametre E-region irregularities. However, a major stumbling block in our understanding of the observations has been an inability to determine accurately the convection electric field $E$ that drives the instability processes, due to a lack of suitable supporting instrumentation within the field-of-view (see also Milan et al., 2003). Overpassing, low Earth orbit (LEO) satellites can help here, but favourable conjunctions are rare. We show in what follows that in the absence of actual measurements of the electric field, we can infer the latitudinal variation of $E$, if not its exact magnitude, using optical observations and applying suitable assumptions. This gives a first stab at placing E-region echo characteristics within the context of the field-aligned current, ionospheric conductivity, and electric field background. Furthermore, the present observations are not unique, Milan et al. (2001) also having reported a double arc feature associated with latitudinally-separated regions of positive and negative Doppler shift echoes. This suggests that an understanding of the present interval will shed light on a not uncommon auroral phenomenon.

Our proposed model of the observations is based on several assumptions, which are detailed below.

1. The arc pair can be treated as two L-shell aligned features, such that a geomagnetic meridian (for arguments sake, the ASC meridian) can be treated as the sole horizontal dimension of the problem. This is a common assumption made when interpreting magnetic field perturbations associated with FAC structures measured by polar-orbiting LEO satellites; if FAC structures are considered to extend to infinity in the cross-track direction then only the along-track dimension is of importance. In reality, our observations show that the auroral forms meet to the west of the ASC meridian, but this does not seem to overly affect our predictions.

2. Each arc, being produced by precipitating electrons, is a sheet of upward FAC. Typically, significant auroral luminosities are associated with current densities of the order of, or in excess of, $1 \mu \mathrm{Am}^{-2}$. To provide current closure there must be an accompanying downward FAC, which we assume lies between the upward FAC sheets, that is in the gap between the arc-pair. The upward and downward FACs are closed through the ionosphere by Pedersen currents flowing in the E-region. We assume that the system is closed, that is no Pedersen currents flow into or out of the system at the poleward or equatorward boundaries. For illustrative purposes we assume that the system is symmetrical, the two arcs being equal in latitudinal width $(250 \mathrm{~km})$ and carrying the same FAC density $\left(1 \mu \mathrm{A} \mathrm{m}^{-2}\right)$. We also assume that the gap is twice as wide as each arc, so the associated current density is equal to that in either arc. Thus, in total, the arc system is $1000 \mathrm{~km}$ wide along the meridian, consistent with the observations of Fig. 3.

3. The electron density of the E-region is enhanced by the precipitation associated with the arcs, such that the arcs are regions of elevated Pedersen conductivity, whereas the gap is of lower conductivity. We assume that the background conductance (height-integrated conductivity) is $3 \mathrm{mho}$, and the elevated conductance is $10 \mathrm{mho}$. Such values are typical for sub-auroral and auroral latitudes, that is for a background and a precipitationenhanced E-region, respectively; conductances can range as high as 30 mho under thin intense arcs (e.g. Milan et al., 1999).

Then, having assumed a latitudinal FAC profile and a conductivity profile it is straightforward to determine the convection electric field necessary to drive Pedersen currents to 
Table 1. Parameters of the arc systems used in the illustrative model (Fig. 4) and the fit to observations (Fig. 5). Parameters indicated are latitudinal width $\Delta d(\mathrm{~km})$, field-aligned current density $j_{\|}\left(\mu \mathrm{Am}^{-2}\right)$, and ionospheric Pedersen conductance $\Sigma_{P}(\mathrm{mho})$

\begin{tabular}{llll|lll|lll}
\hline & Arc 1 & \multicolumn{3}{c|}{ Gap } & \multicolumn{3}{c}{ Arc 2 } \\
\hline & $\Delta d$ & $j_{\|}$ & $\Sigma_{P}$ & $\Delta d$ & $j_{\|}$ & $\Sigma_{P}$ & $\Delta d$ & $j_{\|}$ & $\Sigma_{P}$ \\
\hline Model & 250 & -1 & 10 & 500 & 1 & 3 & 250 & -1 & 10 \\
Fitted & 200 & -1.1 & 9 & 425 & 1.2 & 3 & 250 & -1.1 & 8.1 \\
\hline
\end{tabular}

close the system. Figure $4 \mathrm{a}$ shows the current system envisaged, and the panels below, in order, show the FAC magnitude $j_{\|}$(positive downwards), the E-region Pedersen conductance $\Sigma_{P}$, the height-integrated Pedersen current density $J_{P}$, the polewards electric field $E_{x}$, the magnetic field perturbation associated with the FAC system $b_{y}$, and the ionospheric plasma drift $V_{y}$. All are a function of $x$, the distance polewards along the meridian from the equatorwards edge of arc 1 . In our coordinate system $y$ is positive eastwards, and $z$ points downwards along the magnetic field direction $\boldsymbol{B}$, assumed to be vertical. The model parameters are summarized in Table 1.

Figure $4 \mathrm{~b}$ shows the variation of $j_{\|}$with $x$, indicating two regions of upward FAC on either side of a region of downward FAC. By current continuity, the Pedersen current must be the integral of $j_{\|}$, that is

$J_{P}=\int_{0}^{x} j_{\|} d x$,

assuming no Pedersen current flows equatorwards of the arc system. If upward and downward FACs are of equal overall magnitude, then no Pedersen current is present polewards of the arc system either, as is the case in the present model, Fig. 4 d. From this we can derive the electric field which is necessary to drive this current, as

$J_{P}=\Sigma_{P} E_{x}$

or, from Eq. 1,

$E_{x}=\frac{1}{\Sigma_{p}} \int_{0}^{x} j_{\|} d x$

The variation in $E_{x}$ is shown in Fig. 4e. Before comparing this with our observations, we determine, for interest, the magnetic perturbation that would be measured by a satellite $\mathrm{S}$ passing through the arc FAC structure along the meridian. Representing the FAC density as $\boldsymbol{j}=j_{\|} \hat{z}$, we can determine the magnetic perturbation $\boldsymbol{b}=b_{x} \hat{\boldsymbol{x}}+b_{y} \hat{\boldsymbol{y}}+b_{z} \hat{z}$ from Ampére's Law

$\nabla \times \boldsymbol{b}=\mu_{0} \boldsymbol{j}$,

which gives

$\frac{\partial b_{y}}{\partial x}-\frac{\partial b_{x}}{\partial y}=\mu_{0} j_{\|}$.
From assumption (1) $\partial$ / $\partial y=0$, so Eq. (5) reduces to

$\frac{\partial b_{y}}{\partial x}=\mu_{0} \dot{j}_{\|}$,

and the perturbation is found to be

$b_{y}=\mu_{0} \int_{0}^{x} j_{\|} d x$.

Hence, the magnetic deflection occurs only in the crosstrack direction, and, if the ionospheric Pedersen conductivity were uniform, the deflection would be proportional to the along-track electric field $E_{x}$ (see Eq. 3). The model perturbation is shown in Fig. 4f. A negative or positive gradient in $b_{y}$ with increasing latitude indicates upward or downward FAC, respectively. The magnitude of the $b_{y}$ perturbation predicted is of the order of several $100 \mathrm{nT}$, which is consistent with observations of spacecraft passing through large-scale FAC systems (e.g. Iijima and Potemra, 1976).

Of most interest here is the electric field variation, which is directly associated with the F-region plasma drift $\boldsymbol{E} \times \boldsymbol{B}$ / $B^{2}$ (Fig. $4 \mathrm{~g}$ ), which is directed east-west, with velocity

$V_{y}=\frac{1}{B \Sigma_{p}} \int_{0}^{x} j_{\|} d x$

where we take $B$ to have a value of $5 \times 10^{4} \mathrm{nT}$. The latitudinal variation of $V_{y}$ is as follows. Below arc 1 no plasma drift is expected. Through arc 1 eastwards plasma drift increases in magnitude to several $100 \mathrm{~m} \mathrm{~s}^{-1}$, with a jump to over $1 \mathrm{~km} \mathrm{~s}^{-1}$ at the poleward edge due to the drop in conductivity. Across the arc gap $V_{y}$ turns from positive to equally negative, with a drop in magnitude to several $100 \mathrm{~m} \mathrm{~s}^{-1}$ at the equatorward edge of arc 2, coincident with the increase in conductivity. The magnitude of the westward plasma drift decreases to zero towards the poleward edge of arc 2. Clearly, our situation is idealized, and such sharp boundaries between the different flow regimes would not be expected in reality, but the form of $V_{y}$ gives a prediction that can be tested against our observations.

To this end, the upper panel of Fig. 5 shows the latitudinal variation of Doppler shift measured along beams 0 to 3 of the radar during the scan of 03:43:34 UT, shown in Figs. 1 and 3. To convert Doppler shift to $V_{y}$, the values have been scaled by $-\sec 50^{\circ}$, to account for the fact that the beams point at an angle of roughly $50^{\circ}$ east of magnetic north and Doppler shifts are by convention positive towards the radar and negative away from the radar. In this conversion we implicitly 


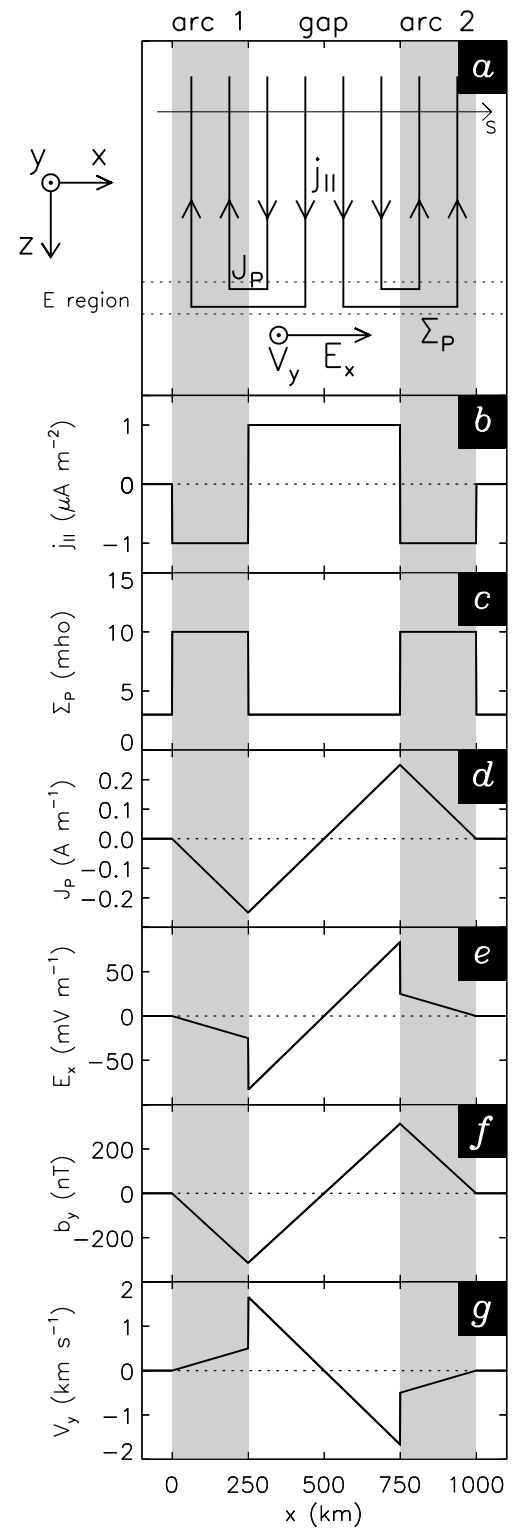

Fig. 4. A model of the expected field-aligned current (FAC) and electric field structure in the observed double-arc feature. (a) A schematic of the current flow in a coordinate system such that $x$ points northwards along the magnetic meridian, $y$ points eastwards, and $z$ points vertically downwards along the magnetic field direction. Grey bars indicate the location of upward FAC, associated with electron precipitation, and hence, auroral luminosity. $(\mathbf{b}-\mathbf{g})$ The latitudinal variation of field-aligned current density $j_{\|}$, Pedersen conductance $\Sigma_{P}$, Pedersen current $J_{P}$, electric field $E_{x}$, the magnetic perturbation experienced by a spacecraft $S$ passing through the FAC system $b_{y}$, and the ionospheric plasma drift $V_{y}$.

assume that the flows measured by the radar are directed zonally (see assumption (1) above). Superimposed is a dashed curve of the same general shape as the prediction of $V_{y}$ in Fig. 4g; peak magnitudes and the distance scale have been tweaked to achieve a fit with the data, but by no more than $10 \%$ and $15 \%$, respectively. The arc parameters required to produce this fit are shown in Table 1. We have marked on the locations of arcs 1 and 2 (grey bars) determined from the $V_{y}$ prediction, and find that this matches closely the co-located WIC auroral observations shown in the lower panel of Fig. 5. Finally, a vertical dashed line has been superimposed which shows the approximate location of the gap between E- and F-region backscatter.

We find that the prediction of $V_{y}$ qualitatively reproduces the observations, that is low positive $V_{y}$ associated with arc 1 , low negative $V_{y}$ associated with arc 2, and high positive and negative $V_{y}$ in the equatorward and poleward portions of the arc gap, respectively. It is always problematic using E-region echoes to infer electric field as it is well known that there is not necessarily a linear correlation between the electric field and Doppler shift. However, that there is also close agreement with the F-region observations supports our picture. This suggests that our assumptions regarding the location of FAC structures relative to the regions of auroral luminosity are correct to a first order, lending strength to our assertion that the arc gap is associated with downward field-aligned current. This is the first time in our studies of E-region backscatter that we have been able to state with confidence the exact location of downward FAC relative to auroral luminosity features (cf. Milan et al., 2001, 2002), allowing us to determine the echo characteristics associated with downward current flow.

Within the E-region backscatter, co-located with the arc gap (between ranges of 400 and $600 \mathrm{~km}$ ), is a mixture of high and low Doppler shift echoes. This TWOPEE E-region echo population has been described in detail by Milan et al. (2003), and a tentative association between it and downward FAC structures was drawn by Milan et al. $(2001,2002)$. We can now make this association more strongly. Villain et al. $(1987,1990)$ described the high Doppler shift component of such echoes in terms of electrostatic ion cyclotron waves excited in the presence of thermally upwelling electrons carrying downward FAC. While some doubt has been cast on this interpretation (Milan et al., 2003), the link with downward FAC appears concrete. We quickly reiterate here the findings of Milan et al. (2003) that the electric field in the vicinity of these echoes appears sufficient to produce the high Doppler shifts observed (contrary to the suggestion of Villain et al., 1987, 1990), and that it is the low Doppler shift component that is depressed significantly below expectations, to approximately $10 \%$ of the high Doppler shift echoes.

Associated with arc 1 we find E-region echoes with Doppler shifts which vary in concert with the predicted driving electric field. As these echoes have Doppler shifts below the ion-acoustic speed, we suggest that they are scattered from gradient drift waves. Without actual electric field measurements we cannot determine the exact relationship between the Doppler shift and $E_{x}$, but we can at least infer that this is close to proportionality.

Finally, we note that a gap in the backscatter is observed near a range of $600 \mathrm{~km}$ (e.g. Fig. 2e). One explanation is that this range corresponds to a transition from E-region to F-region echoes, governed by the altitude at which the radar beams can achieve orthogonality with the magnetic field. In 


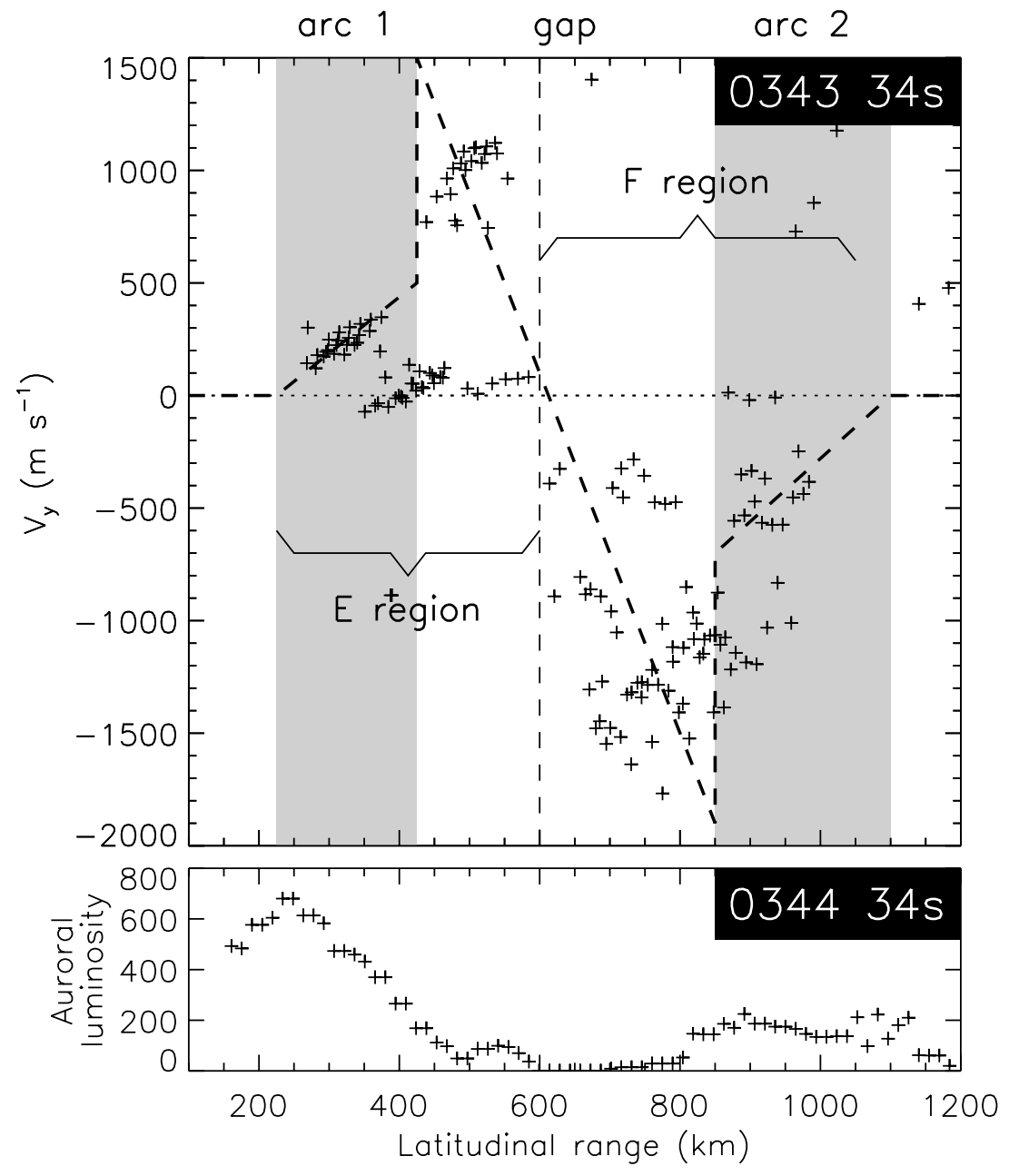

Fig. 5. (Upper panel) The plasma drift $V_{y}$ inferred from the Doppler shift measurements along beams 0 to 3 of the radar during the scan starting 03:43:34 UT. The dashed curve indicates a fit of $V_{y}$ from the model. Superimposed are grey bars indicating the location of arcs suggested by the $V_{y}$ fit. (Lower panel) The auroral luminosity observed by IMAGE FUV/WIC along beam 0 of the radar at 03:44:34 UT. addition, however, the predicted electric field within this region is close to $0 \mathrm{mV} \mathrm{m}^{-1}$ (Fig. 5), in which case irregularities are not expected to be generated (e.g. Milan et al., 1999).

\section{Conclusions}

Coherent HF radar measurements of $\mathrm{E}$ and F-region backscatter from the postmidnight sector suggest a close relationship between echo characteristics and a pair of auroral luminosity features. Assumptions regarding the fieldaligned current structure associated with the arc system allow the latitudinal variation in the convection electric field to be predicted, these predictions matching the observed echo Doppler shifts well. Thus, we can place the E-region backscatter characteristics within the context of the FAC structure, allowing several conclusions to be drawn:

1. Milan and Lester (2001) described how E-region echoes often appear delineated into L-shell-aligned "channels" in which the echo characteristics differ, but fall into distinct populations. These channels appear to be associated with changes in field-aligned current structure, ionospheric conductivity, and the driving electric field, that is a relationship with visual auroral forms is expected, as also investigated by Milan et al. (2000, 2001, 2002).

2. Mixed high and low Doppler shift echoes (TWOPEEs), as described by Milan et al. (2003), are associated with regions of downward FAC. Villain et al. (1987, 1990) implicated thermally upwelling electrons in the generation of the high Doppler shift echoes, which is consistent with our observations. However, as suggested by Milan et al. (2003) and verified by the present study, the electric field within these regions is sufficient to explain the observed Doppler shifts, and so "oblique electron streaming" (Villain et al., 1987) is not necessary to provide additional free energy for instability growth. These high Doppler shifts are greatly in excess of the ion-acoustic speed, seemingly ruling out the two-stream instability as the driving mechanism. In addition, the co-located low Doppler shift echoes have anomalously low shifts, of the order of $100 \mathrm{~m} \mathrm{~s}^{-1}$, much depressed below expectations, to approximately $10 \%$ of the lineof-sight component of the electron drift.

3. Within the region of upward field-aligned current asso- 
ciated with arc 1, E-region echoes are observed with Doppler shifts below the ion-acoustic speed, but which appear proportional to the expected electric field, suggesting that such echoes are scattered from gradient drift waves.

Further study will concentrate on actual measurements of electric field and FAC signatures observed by LEO satellites, allowing for a more direct relationship to be drawn between the instability mechanisms operating in the E-region ionosphere and the observed echo characteristics.

Acknowledgements. CUTLASS is supported by the Particle Physics and Astronomy Research Council (PPARC grant no. PPA/R/R/1997/00256), UK, the Swedish Institute for Space Physics, Uppsala, and the Finnish Meteorological Institute, Helsinki. The optical auroral observation project in Iceland was facilitated by the Science Institute, University of Iceland, and supported by Grant in Aid for Overseas Science Survey (grant no. A: 11304029) from the Japanese Society for the Promotion of Science (JSPS).

The Editor-in-Chief thanks J.M. Noël and another referee for their help in evaluating this paper.

\section{References}

Greenwald, R. A., Baker, K. B., Dudeney, J. R., Pinnock, M., Jones, T. B., Thomas, E. C., Villain, J.-P., Cerisier, J.-C., Senior, C., Hanuise, C., Hunsucker, R. D., Sofko, G., Koehler, J., Nielsen, E., Pellinen, R., Walker, A. D. M., Sato, N., and Yamagishi, H.: DARN/SuperDARN: A global view of the dynamics of highlatitude convection, Space Sci. Rev., 71, 761-796, 1995.

Hanuise, C., Villain, J.-P., Cerisier, J.-C., Senior, C., Ruohoniemi, J. M., Greenwald, R. A., and Baker, K. B.: Statistical study of high-latitude E-region Doppler spectra obtained with the SHERPA HF radar, Ann. Geophysicae, 9, 273-285, 1991.

Iijima, T. and Potemra, T. A.: The amplitude distribution of fieldaligned currents at northern high latitudes observed by Triad, J. Geophys. Res., 81, 2165-2174, 1976.

Mende, S. B., Heetderks, H., Frey, H. U., Lampton, M., Geller, S. P., Habraken, S., Renotte, E., Jamar, C., Rochus, P., Spann, J., Fuselier, S. A., Gerard, J.-C., Gladstone, R., Murphree, S., and
Cogger, L.: Far ultraviolet imaging from the IMAGE spacecraft. 1. System design, Space Sci. Rev., 91, 243-270, 2000 a.

Mende, S. B., Heetderks, H., Frey, H. U., Lampton, M., Geller, S. P., Abiad, R., Siegmund, O. H. W., Tremsin, A. S., Spann, J., Dougani, H., Fuselier, S. A., Magoncelli, A. L., Bumala, M. B., Murphree, S., and Trondsen, T.: Far ultraviolet imaging from the IMAGE spacecraft. 2. Wideband FUV imaging, Space Sci. Rev., 91, 271-285, 2000b.

Milan, S. E. and Lester, M.: Spectral and flow angle characteristics of backscatter from decametre irregularities in the auroral electrojets, Adv. Space Res., 23, 1773-1776, 1999.

Milan, S. E., Davies, J. A., and Lester, M.: Coherent HF radar backscatter characteristics associated with auroral forms identified by incoherent radar techniques: a comparison of CUTLASS and EISCAT observations, J. Geophys. Res., 104, 22 591-22 604, 1999.

Milan, S. E., Lester, M., Sato, N., Takizawa, H., and Villain, J.-P.: Investigation of the relationship between optical auroral forms and HF radar E-region backscatter, Ann. Geophysicae, 18, 608617, 2000.

Milan, S. E. and Lester, M.: A classification of spectral populations observed in HF radar backscatter from the E-region electrojets, Ann. Geophysicae, 19, 189-204, 2001.

Milan, S. E., Lester, M., Sato, N., and Takizawa, H.: On the altitude dependence of the spectral characteristics of decametrewavelength E-region backscatter and the relationship with optical auroral forms, Ann. Geophysicae, 19, 205-217, 2001.

Milan, S. E., Sato, N., Lester, M., Yeoman, T. K., Murata, Y., Doi, H., and Saemundsson, T.: The spectral characteristics of E-region radar echoes co-located with and adjacent to visual auroral arcs, Ann. Geophysicae, 20, 795-805, 2002.

Milan, S. E., Lester, M., and Sato, N.: Multi-frequency observations of E-region HF radar aurora, Ann. Geophysicae, 21, 761-777, 2003.

Villain, J.-P., Greenwald, R. A., Baker, K. B., and Ruohoniemi, J. M.: HF radar observations of E-region plasma irregularities produced by oblique electron streaming, J. Geophys. Res., 92, 12 327-12 342, 1987.

Villain, J.-P., Hanuise, C., Greenwald, R. A., Baker, K. B., and Ruohoniemi, J. M.: Obliquely propagating ion acoustic waves in the auroral E-region: Further evidence of irregularity production by field-aligned electron streaming, J. Geophys. Res., 95, 7833-7846, 1990. 